\title{
EFFECTS OF EXPERIENCE ON DECISIONS FROM DESCRIPTION: EMPIRICAL RESULTS
}

Yuh-Jia Chen, Palm Beach Atlantic University, U.S.A.

James E. Corter, Teachers College, Columbia University, U.S.A.

\author{
dx.doi.org/10.18374/JIBE-21-1.3
}

\begin{abstract}
We investigate how repeated trials with experienced outcome feedback affect risk preferences in description-based decisions under risk, and if the observed effects generalize across gain and loss domains. In Study 1, no significant trend in risk-seeking was observed for Gain problems. However, effects of experienced outcome feedback on decisions from description were observed in the Loss domain: participants initially showed intensified risk-seeking, but became less risk-seeking across 100 repeated trials. Participants then experienced a second set of 100 trials with the "reflected" gain or loss problem. In these Set 2 trials, again participants experiencing Loss problems showed a decrease in risk seeking across the 100 trials, while the proportion of risky choices was essentially flat for Gain problems. Initial Set 2 choice proportions showed an "intercept effect" in the direction of increased EV-maximization, suggesting cross-domain generalization of EV-learning. Study 2 sought to test an alternative explanation for this intercept effect: that framing effects can account for the apparent generalization effects observed in Study 1. Results differed for gains and losses: endowment with a large starting gain mimicked the Study 1 observed "intercept effect" of prior Gain trials on initial risk preferences for Loss trials, suggesting that this intercept effect was due to decision framing of Set 2 trials that incorporates prior gains. But endowment with a large starting loss did not have a significant effect.
\end{abstract}

Keywords: repeated decisions; decisions from experience; risky choice; risk-seeking; EV-learning 